\title{
Parameters of the Dark Halo distribution around M 31
}

\author{
P. Tenjes ${ }^{1}$ and J. Einasto \\ Tartu Observatory, Tóravere, 61602 Estonia
}

\begin{abstract}
Available observational data allow us to discriminate between the visible matter and the dark matter in M 31 and thus to determine the most important parameters of the dark halo (the mass, the radius and the outer extent).
\end{abstract}

\section{Introduction}

In order to shed light on the distribution of visible and dark matter (DM) two approaches are used.

- By using a large sample of galaxies and constructing approximate models with several simplifying assumptions (e.g. spherical bulge, infinitely thin disk, maximal disk hypothesis) and one or two kinds of observations. When averaging the model parameters over a large sample final conclusions may be rather insensitive with respect to the original assumptions (e.g. Persic et al. (1996), Courteau \& Rix (1999)).

- Another possibility is to concentrate on detailed modeling of well-observed individual galaxies. When using many different kinds of observations (up to direct measurements of the stellar composition or line-of-sight velocity profiles) it is possible to abandon many of the simplifying assumptions and thus to obtain more realistic results for these galaxies (e.g. Bottema \& Gerritsen 1997, Rix et al. 1977, Gerhard et al. 1998, Corsini et al. 1999).

It is clear that both methods complement each other and are equally fruitful. Unfortunately in both cases the observational constraints are insufficient to determine all the parameters of DM distribution. On the basis of rotation curves and surface photometry of galaxies it is not possible to determine uniquely the density distribution parameters of the dark halo (total mass, radius and outer extent). As a rule rotation curves remain flat up to the outermost measured points. Thus it remains unknown what the relative contribution of the visible and the dark matter is to the measured rotational velocities. Also, due to the limited extent of the measured rotation curves the extent of dark halos themselves remains unknown.

\footnotetext{
${ }^{1}$ Institute of Theoretical Physics, Tartu University, Tähe 4, Tartu, 51010 Estonia
} 
In this sense the Andromeda galaxy is a fortunate exception. In the present paper we demonstrate that when using additional data it is possible to determine the DM distribution parameters and to calculate the ratio of $M_{\text {dark }} / M_{\text {visible }}$.

\section{A special case: $\mathbf{M} 31$}

For the Andromeda galaxy three kinds of additional information are available.

Firstly, there is the kinematics of the Local Group. Analyzing the relative motion of the Milky Way (G) and the Andromeda galaxy (A) Einasto \& LyndenBell (1982) gave quite strict limits for the total mass of these two galaxies. From their Fig. 5 we derive $M=M_{G}+M_{A}=(4.7 \pm 0.8) \times 10^{12} M_{\odot}$ (for an assumed age of $15 \mathrm{Gyr}$ ). A similar value results from modeling of the kinematics of nearby galaxies by Peebles (1995), where orbits of nearby galaxies are consistent with their observed velocities when the mass of the Milky Way is $M_{G}=2 \times 10^{12} M_{\odot}$ and M 31 has $M_{A}=2.6 \times 10^{12} M_{\odot}$. A smaller mass for the Local Group was derived in a recent study by Courteau \& van den Bergh (1999): $M_{A}+M_{G}=$ $(2.6 \pm 0.8) \times 10^{12} M_{\odot}$ and $M_{A} / M_{G}=1.5$. Thus, both the higher $\left(2.7 \times 10^{12}\right.$ $\left.M_{\odot}\right)$ and the lower $\left(1.6 \times 10^{12} M_{\odot}\right)$ masses will be considered here.

Secondly, we can observe the rotation curve of M 31 at distances where the contribution of the dark matter is already significant $(R \approx 25-40 \mathrm{kpc})$. The surface brightness distribution measurements extend up to galactocentric distances $R \approx 30 \mathrm{kpc}$ (Pritchet \& van den Bergh 1994), direct stellar content observations up to $45 \mathrm{kpc}$ (Durrell et al. 1994, Couture et al. 1995 and Holland et al. 1996). When using these data, we can calculate from chemical evolution models (Worthey 1994,1996 ) the $M / L_{B}$ ratio of visible matter and subtract its contribution from the total mass density. In fact this gives us a quite good estimate for the dark matter (DM) density in particular regions, e.g. the central density we derive is $\rho_{D M}(0)=0.057 M_{\odot} \mathrm{pc}^{-3}$. The central density of a spherical subsystem is $\rho(0)=\kappa M /\left(4 \pi a_{c}\right)^{3}$, where $a_{c}$ is the core radius and $\kappa$ is a normalizing constant ( $\kappa$ depends on the density distribution law, and in the case of truncated isothermal sphere on the tidal radius). Hence, in order to determine all the density distribution parameters of the DM we need one more constraint.

Thirdly, a constraint can be obtained from the mass density in the galactic plane. For M31 we have independent determinations of the H I gas layer thickness and the gas velocity dispersion. According to the well-known formula $\rho=\sigma_{g}^{2} /\left(2 \pi G z_{0}^{2}\right)$, where $\sigma_{g}$ is gas velocity dispersion and $z_{0}$ is gas layer thickness, we can calculate the mass density in galactic plane. At $R=8 \mathrm{kpc}$, where the contribution of the bulge is negligible and the dominating contribution to this density is due to the old stellar disk and the dark matter, $\sigma_{g}=32 \mathrm{~km} \mathrm{~s}^{-1}$ (Emerson 1976), $z_{0}=0.7 \mathrm{kpc}$ (Whitehurst et al. 1978). Thus the total mass density in the galactic plane at a radius of $8 \mathrm{kpc}$ is $\rho_{T}=0.08 M_{\odot} \mathrm{pc}^{-3}$ (see e.g. Tenjes et al. 1994).

\section{Results}

Taking into account all these restrictions we believe that we can realistically model the dark and visible matter distributions in M 31 . For $M_{A}=2.7 \times 10^{12}$ 


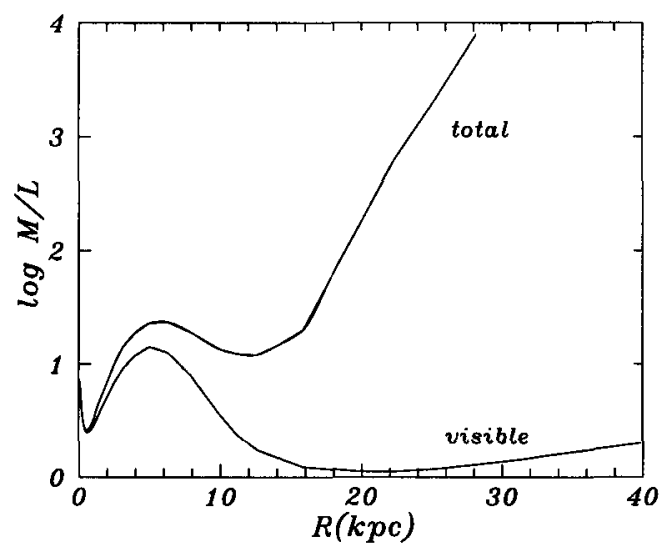

Figure 1. The local mass-to-light ratios of visible and dark matter in $\mathrm{M} 31$.

$M_{\odot}$ the outer extent of the dark halo is $400 \mathrm{kpc}$, the core radius $a_{c}=3.8 \mathrm{kpc}$ (the harmonic mean radius $a_{0}=60 \mathrm{kpc}$ ). For $M_{A}=1.6 \times 10^{12} M_{\odot}$ the outer extent is $340 \mathrm{kpc}$, the core radius $a_{c}=3.2 \mathrm{kpc}$ (the harmonic radius $a_{0}=50$ kpc. Neither of this models have a so-called "maximum disk". We conclude that for individual galaxies the dark and visible matter contributions must be individually studied and an optimum disk must be chosen. (As an example, for M 81 a maximum disk gives the best fit, see Tenjes et al. 1998.)

The ratio of DM mass to the mass of visible matter on the scale of $\sim 0.5$ $\mathrm{Mpc}$ is $\approx 20$ to $\approx 35 M_{\odot} / L_{\odot}$.

We can calculate local mass-to-light ratios by dividing the mass and luminosity in a shell limited by the radius $R$ and $R+\Delta R$. Resulting local $M / L_{B}$ ratios as a function of $R$ for the first model (larger dark halo) are given in Figure 1 . At the greatest distances where surface brightness observations have been carried out (Pritchet and van den Bergh 1994) the local $M / L$ ratio is evidently $>1000 M_{\odot} / L_{\odot}$.

Acknowledgments. P. T. acknowledges financial support provided by the Academy of Finland and the Estonian Science Foundation (grant 2627).

\section{References}

Bottema, R., \& Gerritsen, J. P. E. 1997, MNRAS, 290, 585

Courteau, S., \& Rix, H.-W. 1999, ApJ, 513, 561

Courteau, S., \& van den Bergh, S. 1999, AJ, (in press)

Couture, J., Racine R., Harris, W. E., \& Holland, S. 1995, AJ, 109, 205

Durrell, P. R., Harris, W. E., \& Pritchet, C. J. 1994, AJ, 108, 2114

Einasto, J., \& Lynden-Bell, D. 1982, MNRAS, 199, 67

Emerson, D. T., 1976, MNRAS, 176, 321 
Gerhard, O., Jeske, G., Saglia, R. P., \& Bender R. 1998, MNRAS, 295, 197

Holland, S., Fahlman, G. G., \& Richer, H. B. 1996, AJ, 112, 1035

Peebles, P. J. E., 1995, ApJ, 449, 52

Persic, M., Salucci, P., Stel, F. 1996, MNRAS, 281, 27

Pritchet, C. J., \& van den Bergh, S. 1994, AJ, 107, 1730

Rix, H.-W., de Zeeuw, P. T., Carollo, C. M., Cretton, N., \& van der Marel, R. P. 1997, ApJ, 488, 702

Tenjes, P., Haud, U., \& Einasto, J. 1994, A\&A, 286, 753

Tenjes, P., Haud, U., \& Einasto, J. 1998, A\&A, 335, 449

Whitehurst, R. N., Roberts, M. S., \& Cram, T. S., 1978, in Structure and properties of nearby galaxies, IAU Symp. 77, E. M. Berkhuijsen \& R. Wielebinski, Dordrecht: Reidel, p175

Worthey G. 1994, ApJS, 95, 107. See also: http://astro.sau.edu/ worthey/dial/dial_a_model.html

Worthey G. 1996, AAS CD, vol. 7, file Worthey.tar 